\title{
Les Pères du désert d'Égypte : utopie et silence
}

Jean-Baptiste Bernard

\section{OpenEdition}

Journals

Édition électronique

URL : http://journals.openedition.org/recherchestravaux/555

DOI : 10.4000/recherchestravaux.555

ISSN : 1969-6434

Éditeur

UGA Éditions/Université Grenoble Alpes

Édition imprimée

Date de publication : 30 décembre 2012

Pagination : 121-135

ISBN : 978-2-84310-238-7

ISSN : 0151-1874

Référence électronique

Jean-Baptiste Bernard, «Les Pères du désert d'Égypte : utopie et silence », Recherches \& Travaux [En ligne], 81 | 2012, mis en ligne le 30 juin 2014, consulté le 07 septembre 2020. URL : http://

journals.openedition.org/recherchestravaux/555; DOI : https://doi.org/10.4000/recherchestravaux. 555 
Jean-Baptiste BERNARD

Université Stendhal - Grenoble 3

\section{Les Pères du désert d'Égypte : utopie et silence}

Peut-on parler de mythe à propos des Pères du désert? Si l'on considère l'extraordinaire fortune littéraire, et plus généralement culturelle, qu'ont connue des figures comme celles d'Antoine le Grand, de Macaire, de Pacôme, il semble que oui. Au-delà de l'historicité des personnages, leur rôle de référent culturel majeur en Orient comme en Occident permet d'étudier, à travers eux, outre la formation d'une tradition religieuse toujours vivante, celle d'un mythe, épistémologique et politique.

Le champ d'étude est vaste, et il ne s'agira ici que d'en esquisser quelques axes. De l'abondante littérature patristique de l'Antiquité tardive, jusqu'aux travaux de redécouverte menés au $\mathrm{xx}^{\mathrm{e}}$ siècle, notamment par les moines des abbayes de Solesmes et de Bellefontaine, c'est tout un imaginaire qui prend corps autour des figures des premiers ermites d'Égypte. Le dernier quart du siècle dernier a vu réactivés les thèmes forts du mythe. La dimension politique, celle du vivre ensemble, est une composante majeure du cours donné par Roland Barthes au Collège de France en 1977. Dans son essai, Les Hommes ivres de Dieu², Jacques Lacarrière s'interroge sur l'expérience spirituelle de la retraite au désert, dans son rapport à la parole, à la folie, tout comme François Weyergans, dans le roman qu'il consacre à l'une des figures fameuses du monachisme égyptien, Macaire le Copte ${ }^{3}$.

Dès les textes antiques, ces thèmes sont présents, et problématisent ce qui nous semble être un mythe qui interroge la capacité de l'homme à vivre

I. R. Barthes, Comment vivre ensemble, Cours et séminaires au collège de France (1976-1977), éd. scientifique de Cl. Coste, Seuil, coll. «Traces écrites», 2002.

2. J. Lacarrière, Les Hommes ivres de Dieu, Fayard, 1975.

3. Fr. Weyergans, Macaire le Copte, Gallimard, I98I. 
en communauté, la façon, aussi, qu'il a de concevoir le rôle de la connaissance et du langage dans sa structure d'individu. Le premier grand texte, Vie et conduite de notre saint Père Antoine ${ }^{4}$, écrite par le patriarche Athanase d'Alexandrie au milieu du Iv ${ }^{\mathrm{e}}$ siècle, pose d'emblée ces questions. Deux décennies plus tard, Jérôme de Stridon, dans sa Vie de Paul de Thèbes, premier ermites, reprend partiellement ces thèmes et leur fait prendre le chemin de Rome et de tout l'Occident chrétien. Au début du ve siècle, ce sont Pallade de Galatie et son Histoire lausiaque, puis Rufin d'Aquilée, son traducteur en latin, qui achèvent de donner aux Pères du désert d'Égypte leur renommée internationale, et entérinent le passage de l'histoire au mythe ${ }^{6}$.

C'est bien de cela qu'il s'agit : en quelques décennies, les fondateurs de groupuscules locaux, profondément enracinés dans leur contexte particulier, provoquent un changement radical qui se mesure à l'échelle d'une civilisation, deviennent, de fait, les référents qui structurent une nouvelle façon de concevoir la vie en société et jusqu'à l'homme lui-même. En se soustrayant aux structures traditionnelles de la société, en se retranchant du monde, les Pères du désert ouvrent une nouvelle page de l'histoire proche-orientale et européenne. Après eux, la question reste ouverte d'une utopie chrétienne de la vie en cellules sociales réduites, reste ouverte aussi la question d'une possible vanité de la raison, qui a nourri toute la tradition monastique et mystique, et n'a eu de cesse d'interroger, voire de critiquer ne serait-ce qu'en l'état de contre-modèle, la philosophie, des diverses écoles médiévales au rationalisme moderne.

Aujourd'hui, les figures de saint Antoine le Grand, Paul de Thèbes, Pacôme, Macaire l'Ancien, pour ne citer que les plus célèbres, sont investies d'une charge symbolique particulièrement forte. Solitaires, les Pères représentent l'anarchisme chrétien, l'expression d'une quête d'absolu si puissante qu'elle interdit le commerce des hommes, et jusqu’à la vie de la chair. En petites cellules retranchées dans le désert, ils proposent de faire de la vie commune une préfiguration du Royaume de Dieu, un îlot de vie véritable dans un monde perçu comme soumis à l'arbitraire du mal. Plongés dans le silence,

4. Nous abrégerons fréquemment en Vie d'Antoine. Notre édition de référence est : Saint Athanase, Antoine le Grand, père des moines, trad. de B. Lavaud, Cerf, coll. "Trésors du christianisme», 2007.

5. Saint Jérôme, Vivre au désert, Vies de Paul, Malchus, Hilarion, trad. de J. Miniac, Grenoble, Éditions Jérôme Millon, 1992. Le titre de la vie de Paul est plus précisément celui que nous avons indiqué, Vie de Paul de Thèbes, premier ermite, nous abrégerons régulièrement en Vie de Paul.

6. Pour le texte de Pallade, signalons une traduction récente : Pallade d'Hélénopolis, Histoire lausiaque, trad. de N. Molinier, Bégrolles-en-Mauge, Abbaye de Bellefontaine, I999. Le texte de Rufin, à notre connaissance, n’a pas été traduit, voir par exemple : Tyrannius Rufinus, Historia monachorum (éd. scientifique de E. Schulz-Flügel), Berlin-New York, De Gruyter, I990. 
ils montrent que toute parole s'annule devant la Parole de Dieu, en somme, que la quête spirituelle est une quête d'anéantissement, que l'individu est une illusion, une créature de l'orgueil humain. Ouvrant la bouche, ils prêchent à rebours de l'intelligence et de la raison, pratiquent l'anti-discours par excellence, celui qui cherche à défaire l'appareil de l'esprit pour que souffle en lui l'altérité de l'absolu. La mythologie chrétienne a volontiers retenu des Pères du désert le pittoresque des attaques démoniaques et les pratiques presque folkloriques des extrémistes, comme Jacques de Voragine dont la Légende dorée, à propos des Pères, est assez peu spirituelle. C'est, nous semble-t-il, négliger la dimension performative du mythe : si le mythe est une "histoire», c'est aussi, et sans doute en premier lieu, l'expression d'un sens possible, une prise en charge et une structuration du désir de vérité; en cela, plus que prédicatif le mythe est un espace d'élaboration, celui de l'évolution et de la création du sens. Ainsi, c'est à ce titre, bien plus qu'à celui du merveilleux ou du répertoire symbolique, que l'histoire des Pères du désert est investie d'une fonction mythique, en particulier par les quatre thèmes que nous évoquions : la rupture par la solitude, puis l'expérience communautaire, l'ascèse du silence et la subversion du discours.

Pourquoi cette importance, cette place à part des Pères du désert dans le paysage intellectuel de l'Occident et de l'Orient chrétiens? Comment l'universalisme chrétien a-t-il pu construire avec une histoire rigoureusement égyptienne un répertoire mythique dans lequel il puisa, jusqu'à nos jours, des exemples fondamentaux dans la définition de son attitude à l'égard de la connaissance et de la cité? C'est certainement la radicalité du propos des Pères, la rupture, le départ au désert, qui est à l'origine de la riche tradition qui découle d'eux, tant elle est problématique, pour le monde et pour la pensée. Pour essayer de saisir cette dynamique, nous nous proposons de revenir aux origines du mythe, égyptiennes et littéraires.

\section{L'Égypte et la spiritualité du désert}

L'Égypte du III $^{\mathrm{e}}$ siècle offre un paysage religieux d'un rare dynamisme : le christianisme y connaît une importante expansion, alors que les grands cultes polythéistes brûlent de leurs derniers feux et que de nombreuses sectes, notamment gnostiques, connaissent un succès parfois éclatant. Les persécutions antichrétiennes organisées par le pouvoir impérial, les luttes doctrinales au sein de la jeune église et les aspirations à une vie spirituelle d'une exigence absolue favorisent l'émergence de nouveaux courants. Avant de nous pencher sur le traitement par les docteurs de l'Église de certains aspects des 
biographies, réelles ou fictives, des Pères du désert, il convient de mettre la démarche de ces derniers en perspective.

Le mouvement érémitique apparaît dans un premier temps comme la continuation, sous un autre étendard idéologique, d'une pratique religieuse propre à certains cultes du polythéisme égyptien, en particulier celui de Sérapis. Ce dieu relevait d'une construction syncrétique voulue par Ptolémée, le premier pharaon de la dynastie Lagide : il réunissait des traits d'Osiris, d'Apis, de Zeus, d'Hadès. Son culte, sous la forme d'un taureau, connut une fortune grandissante dans l'Antiquité tardive, comme d'autres cultes à mystères, ceux, par exemple, d'Isis et de Mithra' ${ }^{7}$. L'aspect de ce culte qui intéresse le plus le mouvement érémitique est l'existence, au Serapeum de Memphis, d'une communauté de prêtres vivant enfermés dans l'enceinte du temple (les katochoi, parfois francisés en "catoques», du grec «reclus», "possédés»). Si l'Antiquité a connu de nombreuses formes de consécration d'un individu à une divinité, ces prêtres sont les premiers, à notre connaissance, à rechercher un idéal de vie solitaire, tourné intégralement vers la contemplation et la méditation, en marge de toute manifestation publique de la religion. Contrairement à d'autres groupes religieux, comme les Esséniens par exemple, les reclus du Serapeum ne visent pas à représenter une forme de communauté parfaite : ils sont tout entiers tournés vers la personnalisation de la relation au sacré. Il faut aussi signaler l'existence, rapportée par Philon d'Alexandrie", d'une secte propre au judaïsme hellénisé, particulièrement florissante en Égypte : les Thérapeutes, dont la vie alternait entre prière solitaire et temps communautaires. Pour de nombreux auteurs, ce mouvement inspira en partie le cénobitisme chrétien, lorsque, devant l'affluence de disciples, les ermites durent élaborer une règle afin de pérenniser l'existence des communautés.

Les ermites, d'abord égyptiens, étaient familiers de cette idée selon laquelle dans la réclusion et la solitude se construit un rapport authentique et personnel à la divinité. Chrétiens, ils transforment la démarche des adorateurs de Sérapis, par l'apport des Écritures. La Bible abonde en figures de solitaires, quittant tout pour le désert, lieu privilégié de la rencontre avec Dieu : Moïse, Élie, Jean-Baptiste, le Christ lui-même pendant les quarante jours de sa tentation, sans oublier le grand patriarche Abraham. Mais dans la Bible, le désert et sa solitude ont avant tout une fonction de transition : aller au désert, c'est

7. Sur les cultes à mystères en général, on consultera avec profit : Y. Lehmann (dir.), Religions de l'Antiquité, PUF, 1999. Sur Sérapis en particulier, la documentation en français est assez rare; on peut, pour l'aspect qui nous intéresse, lire le passage que lui consacre Fr. Daumas dans son introduction au De Vita contemplativa de Philon d'Alexandrie: Philon d'Alexandrie, De Vita contemplativa, trad. de P. Miquel, Cerf, I963, p. 62-66.

8. Il consacre aux Thérapeutes son De Vita contemplativa, voir note précédente. 
chercher la confirmation d'une vocation. Le désert est le lieu de la préparation, de l'initiation : ainsi de l'Exode, qui confirme la vocation d'Israël à endosser le rôle de "peuple élu", représentant, dans ses errements, tous les errements humains, dans sa sainteté, toute la perfectibilité de l'homme. Au niveau individuel, le désert est le lieu où se forme la parole prophétique, celui où le prophète, jusqu'au Christ, assiste à la genèse, en lui, du verbe divin. L'initiation, dans la solitude et l'ascèse, au pouvoir de la parole divine, débouche invariablement sur un retour à l'humanité vers qui cette parole est projetée.

Les ermites ne peuvent prétendre suivre le même parcours initiatique : quand Antoine s'en va au désert, en 269, tout a déjà été révélé. Il s'agit alors de faire la synthèse des héritages biblique et polythéiste : le désert reste le lieu de la rencontre personnelle avec Dieu, mais vidée de la motivation prophétique. Reclus dans le désert - la nature de l'espace désertique garantissant la réclusion -, l'ermite cherche la relation personnelle qu'y ont connue les prophètes et les patriarches, mais selon le même mode que les reclus du Serapeum et les Thérapeutes, celui d'une méditation permanente d'un corpus existant, et non d'une parole délivrée directement par la divinité9. Il faut, bien entendu, nuancer cet aspect : pour l'ermite, si la révélation est pratiquement fixée - nous sommes dans les siècles de définition du canon -, elle n'en sollicite pas moins, en lui, une aptitude unique à la recevoir. La recevoir, et non la comprendre : hormis dans la vie écrite par Athanase ${ }^{\mathrm{ro}}$, l'ermite enseigne plus volontiers par ellipse qu'en exposant une interprétation du texte révélé. L'ermite est avant tout, dans sa relation au corpus biblique, celui qui cherche non à comprendre mais à entendre, à mettre en œuvre, non à gloser. Il est le récepteur de la parole révélée dans son dynamisme signifiant : il entre en résonance, il se consacre au silence et à la prière brève, pour ne pas interférer intellectuellement avec l'altérité absolue qui se manifeste dans la parole qu'il médite en permanence (la «rumination» des textes bibliques).

Cette vocation de l'ermite, bien qu'elle opère une synthèse originale du point de vue judéo-chrétien et de la tradition locale, se définit néanmoins spécifiquement en regard du référent égyptien. En effet, le rôle de l'Égypte dans la Bible, d'une part, et, de l'autre, le contexte historique entourant les premiers ermites sont déterminants dans la construction du projet des Pères.

9. Les quelques fois où Dieu, par des anges ou des visions, s'adresse aux ermites, il s'agit de les conforter et de les conseiller, non de leur révéler une vérité complémentaire de celle de la Bible. Ainsi, par exemple, d'une vision d'Antoine (Athanase, Vie d'Antoine, ouvr. cité, p. 19). Dans un autre passage, Antoine enseigne aux moines les signes qui permettent de différencier visions angéliques et visions démoniques (ibid., p. 46-48).

Io. Une proportion importante du récit est consacrée aux enseignements d'Antoine, d'une façon clairement didactique (ibid., p. 27-58). 
La terre d'Égypte, dans la tradition judéo-chrétienne, a un rôle ambivalent. Terre de l' «abomination païenne», de la tentation, de l'oppression, elle est aussi terre de confirmation. Le peuple d'Israël, en Égypte, est tour à tour réduit en esclavage, persécuté pour sa différence et tenté par le paganisme, avant de suivre l'appel de Yahvé. Il en va de même pour certains personnages importants de la geste biblique : Joseph, fils de Jacob, et surtout Moïse. Pour le peuple élu, sans cesse à la recherche de son identité, de ses institutions et de son territoire, l'Égypte représente un miroir inversé, le paradigme antithétique de sa propre vocation, sans commune mesure avec d'autres peuples, comme les Philistins, bien qu'ils jouent un rôle comparable.

Sans entrer en profondeur dans les aventures des patriarches comme séquences d'un même récit de l' «origine», il importe d'avoir à l'esprit cette équation fondamentale : l'Égypte est, pour Israël, la possibilité de s'assimiler au monde, le contre-modèle à la fois séduisant et repoussant qui nécessite la différenciation radicale d'Israël vis-à-vis des autres peuples. Ainsi, c'est le référent égyptien qui structure l'opposition entre Révélation et religion qui est à l'origine de la recherche de la "Terre promise», dans l'Exode. Pour les juifs puis pour les chrétiens, il s'agit, après la sortie d'Égypte, de suivre un Dieu qui parle, qui se révèle par la bouche des prophètes puis du Christ, et se révèle essentiellement pour parler des relations possibles avec lui, au contraire de la religion, perçue dans la Bible comme un culte figé dans des catégories morales et des marchandages propitiatoires, dont l'Égypte pharaonique, avant d'autres peuples, est le parangon. Les ermites sont particulièrement sensibles à cette dynamique de la Révélation, parole qui tranche les constructions spéculatives et les rituels humains, au point de rompre avec leur humanité pour mieux l'entendre au désert.

Cette référence culturelle, dans l'église primitive, va de pair avec le contexte immédiat qui entoure son développement. Si ce sont désormais les Grecs, et surtout l'Empire romain, qui représentent le modèle d'humanité auquel il faut se soustraire, l'Égypte est la province où celui-ci se manifeste avec le plus de brutalité, selon les auteurs patristiques : les persécutions de Dèce, de Valérien, d'Aurélien puis de Dioclétien, entre 250 et 303, auraient particulièrement touché les chrétiens du nord de l'Afrique, et joué un rôle important dans le mouvement érémitique, qui correspondrait entre autres à une volonté de se mettre à l'abri des persécutions. Cela dit, Antoine, dans le récit d'Athanase ${ }^{\text {II }}$, déplore de ne pas trouver le martyr, et d'autres textes de l'église primitive, comme les épîtres de Clément de Rome ou le récit connu sous le titre de Martyre de Polycarpe, montrent à quel point le martyre était valorisé, voire

II. Ibid., p. 58 . 
désiré dans les communautés chrétiennes ${ }^{12}$. Plus, alors, qu'un refuge, le désert représente le lieu d'une rupture avec la société : entre persécutions et référent biblique, le désert d'Égypte apparaît comme le lieu absolument unique - au moins au début -, où cette rupture est possible.

Si bien des formes de pratiques spirituelles radicales ont vu le jour dans l'église primitive, célibat consacré, communautés de vierges, voire castration, l'apparition de l'érémitisme, avec saint Antoine, répond au caractère unique du contexte égyptien. D'une part, l'héritage du culte de Sérapis et des Thérapeutes et sa mise en regard des figures bibliques du désert nourrit les expériences menées par les ermites puis par les premières communautés cénobitiques. D'autre part, la charge symbolique de l'Égypte dans la psyché collective des communautés judéo-chrétiennes et son actualisation, dans les persécutions romaines, comme condensé de tous les aspects de l'humanité que le chrétien doit refuser joue un rôle fondamental dans le désir de rupture incarné par les anachorètes. Ces différents facteurs, nous semblet-il, montrent l'unité et l'originalité du parti des Pères, Antoine en premier, comme directement lié à l'imaginaire et à l'histoire de l'Égypte, lieu de passages permanents entre le monde antique et la "nation élue», entre la spécificité de la Révélation et les cultures religieuses qui l'environnent et parfois la conditionnent.

Avant même que les premiers ermites ne se lancent dans le pari de rencontrer et de fréquenter Dieu au désert sans vouloir en tirer enseignement doctrinal ni structure de pouvoir, l'Égypte offrait aux spéculations religieuses une matière multiple et des exemples de réalisation très nombreux. Antoine au désert, et avec lui combien d'autres, l'Égypte apparaît comme le lieu d'élaboration d'une synthèse unique dans l'histoire religieuse, qui débouche sur un modèle aujourd'hui répandu et décliné de multiples façons dans les christianismes d'Orient et d'Occident. De l'unicité d'une histoire locale à un phénomène spirituel et social qui a rapidement débordé son cadre méditerranéen, il y a une étape fondamentale : l'écriture, par les pères et docteurs de l'Église, de textes qui posent l'érémitisme comme porteur d'une problématique universelle, en premier lieu les Vies écrites par Athanase et Jérôme ${ }^{13}$.

I2. Sur cette question, voir la première épître de Clément de Rome aux Corinthiens et le Martyre de Polycarpe, dans Les Pères apostoliques, trad. de Fr. Quéré, Seuil, I980.

I3. Athanase, Vie d'Antoine, ouvr. cité; Jérôme, Vie de Paul, ouvr. cité. 


\section{Érémitisme et utopie monachique}

Du vivant des Pères du désert, déjà, se constituait un corpus important, à partir duquel le mythe allait prendre son essor. D'un côté, les Vies que nous avons mentionnées, de l'autre, des recueils de sentences, d'apophtegmes. Ces recueils, d'abord en copte et en grec, se diffusèrent rapidement dans tout l'Orient chrétien, contribuant significativement à la bonne fortune des modèles érémitiques et monachiques égyptiens. Les apophtegmes, construits sur le modèle de la transmission orale, "Abba untel disait, Abba Antoine m'a dit», constituent un ensemble très vaste, où s'expriment remarquablement les aspects les plus intéressants de la problématique qui nous occupe : relations aux maîtres, aux disciples, aux visiteurs, au pouvoir, pratique de l'ascèse intellectuelle, du silence. Cela dit, ces recueils étaient destinés essentiellement aux religieux, souvent déjà chevronnés, afin de leur donner matière à méditation et à révision de leurs propres pratiques. Le mythe s'élaborait ailleurs, dans les discours produits par les docteurs de l'Église et destinés à la société séculière : en parlant de ces Pères qui ne voulaient pas qu'on parle d'eux, ils ont si bien montré l'originalité de leur charisme qu'aucune société chrétienne n’a plus jamais pu se penser sans eux.

De fait, la spécificité des Vies écrites par Athanase et Jérôme, puis par Pallade ${ }^{14}$, peu de temps après la mort des Pères fondateurs (saint Antoine meurt vers l'an 356), tient dans leur projet littéraire : orientées vers le monde, elles répondent à un parti de vulgarisation et de prédication. Elles visent un lectorat qui a à intervenir dans les affaires du monde : notables chrétiens, clergé séculier. Or, la fin du III $^{\mathrm{e}}$ siècle et les décennies suivantes, marquées par les querelles théologiques et le changement de statut des chrétiens dans l'Empire, constituent une période de définition pour l'Église : elle doit fixer définitivement sa doctrine, passer d'un fonctionnement collégial qui convenait à des communautés dispersées à une structure plus forte, à même de maintenir sa cohésion d'institution en passe de devenir officiellement la religion d'État. Les mouvements érémitiques et monachiques appellent ainsi à être modérés et contrôlés de plus près, afin que ces tentatives chrétiennes d' "anarchie» ou d'«utopie» ne soient pas un frein, du fait de leur projet subversif, à la christianisation de l'Empire.

C'est bien à ce niveau que les textes d'Athanase et de Jérôme interviennent, dans un premier temps. Dans la Vie de saint Antoine d'Athanase, ce débat est particulièrement visible, quoique mené non sans subtilité : sans cesse, l'évêque

I4. Voir les références complètes en notes 4,5 et 6. 
d'Alexandrie cherche à lier l'extrême rigueur d'une quête personnelle et profondément asociale aux structures ecclésiales, notamment celle qu'il incarne, l'épiscopat, puis, par extension, à un État chrétien ${ }^{15}$. Athanase est à la croisée de deux principes, posés par Jean et l'apôtre Paul : d'une part, le chrétien est "dans le monde sans être du monde ${ }^{16}$ », d'autre part, le chrétien doit considérer le pouvoir en place comme voulu par Dieu ${ }^{17}$.

Antoine l'Ermite est, de par sa trajectoire personnelle, le personnage dans lequel Athanase peut trouver une forme de réconciliation de ces deux principes, en apparence opposables. Ainsi, Antoine est bien le représentant de la vocation du chrétien telle que l'ont définie tous les évangélistes, et Jean plus clairement peut-être que les autres : en disciple du Christ, il est «fils de la lumière», appelé à vivre pleinement sa filialité, en contradiction avec l'ordre du monde, fondé sur les illusions du pouvoir et de la chair. Antoine, au désert, accomplit la plénitude de cette filialité : pour vaincre les démons et se soustraire à la possibilité d'exercer du pouvoir dans la communauté, il se fait vulnérable, pauvre et nu. Ses forces ne sont que de Dieu : la grâce qui le protège et la prière qui le soutient, qui fait de son ascèse un dialogue avec Dieu, et non une discipline de perfectionnement personnel. Antoine au désert ne recherche pas la sainteté mais le salut : dans la fréquentation perpétuelle de son Père, par la prière et les Écritures, il rompt avec son humanité pour n'être plus que l'enfant serviteur qui écoute son maitre pour être jugé digne de le rejoindre dans la vie éternelle, non par ses mérites personnels, mais par son attitude.

C'est là le versant contemplatif de la vocation d'Antoine, qui obéit aux exigences formulées dans les évangiles et les épîtres de Jean, entre autres. Mais Antoine est aussi actif, et parfois militant. Nous reviendrons sur ses prédications, puisqu'elles nous permettront d'aborder la mythification des Pères du désert dans sa fonction épistémologique, il convient néanmoins d'aborder en premier les relations d'Antoine avec le pouvoir terrestre.

Ces relations sont de deux ordres, en fonction des époques : d'abord indirectes, elles se définissent par la recherche du martyr. Lors des persécutions, Antoine rejoint Alexandrie pour soutenir la communauté ébranlée, et chercher le salut dans la mort, ultime témoignage du chrétien. Forme extrême de

I5. Voir, par exemple, les chapitres intitulés "Respect d'Antoine pour le clergé», "Horreur d'Antoine pour le schisme et l'hérésie» et "À la demande des évêques, Antoine vient à Alexandrie réfuter les ariens» (Athanase, Vie d'Antoine, ouvr. cité, p. 80-83).

I6. Voir l'Évangile de Jean, XVII, II-I6. Édition de référence : La Bible, Nouveau Testament, trad. de J. Grosjean et M. Léturmy, Gallimard, coll. «Bibliothèque de la Pléiade», I97ı. Voir p. 328 pour le passage en question.

17. Voir saint Paul, Épitre aux Romains, XIII, I-7. La Bible, ouvr. cité, p. 504-506. 
négation du monde, son vœu ne fut pas accompli : appelé à guider les fidèles qui rejoignent en masse le désert, Antoine se voit refuser le martyr, il est, déjà, un guide, et doit mener jusqu'au bout le travail du guide.

Le temps des persécutions terminé, Antoine se voit respecté et même recherché par le pouvoir. Lisons le chapitre 8I de la vie rédigée par Athanase :

La renommée d'Antoine parvint jusqu'aux empereurs. Ayant appris ces choses, Constantin-Auguste, Constance-Auguste et Constant-Auguste lui écrivirent comme à un père, lui demandant de leur répondre. Il n'appréciait guère ces lettres impériales, n’en éprouvait pas de joie, mais restait le même qu'avant de les avoir reçues. Quand on les lui avait apportées, il avait appelé les moines et dit : «Ne vous étonnez pas que l'empereur nous écrive : c'est un homme, mais admirez plutôt que Dieu ait écrit une loi aux hommes et nous ait parlé par son propre Fils.» Il ne voulait pas recevoir les lettres, disant qu'il ne saurait pas y répondre. Mais ses moines le décidèrent parce que, les empereurs étant chrétiens, il ne fallait pas les scandaliser par un affront; il accepta donc qu'on lui lût les lettres. Dans sa réponse, il félicita ses correspondants d'adorer le Christ, leur donna des conseils pour leur salut; ils ne devaient pas faire grand cas des choses présentes, mais penser plutôt au jugement; le Christ seul est roi véritable et éternel. Il leur recommandait d'aimer les hommes, d'observer la justice, d'avoir souci des pauvres. Les princes reçurent ses lettres avec joie. Ainsi était-il chéri de tous. Chacun voulait l'avoir pour père ${ }^{18}$.

La réaction d'Antoine face aux sollicitations de l'empereur et des deux vice-empereurs, ses fils, fait une synthèse pertinente des principes donnés au chrétien dans ses relations au pouvoir : légitime, parce que d'une certaine manière voulu par Dieu, le pouvoir n'en est pas moins par nature contraire à la vocation du chrétien. Antoine rappelle que toute loi reste relative devant l'exigence de l'appel du Christ : «[...] vends tout ce que tu as et donne-le aux pauvres, et viens, suis-moi ${ }^{19} »$. Empereurs ou esclaves, les chrétiens doivent se différencier de leur statut social : ils sont avant tout «enfants de Dieu ", et, à ce titre, ne doivent investir dans leur fonction politique, quelle que soit son échelle, que les commandements de charité et de fraternité. C'est la seule concession qu'Antoine peut faire au monde, le reste appartient au désert, réel ou métaphorique : lieu hors du monde où la personnalité se dépouille pour rencontrer la divinité. C'est une anecdote intéressante du point de vue du mythe, dans la mesure où la synthèse qu'elle opère n'est, au fond, pas une proposition de projet social, mais la définition d'une attitude.

Ainsi, bien qu'Antoine, par ses relations épisodiques avec l'épiscopat et les princes, ne soit plus en conflit ouvert avec la société, il lui est tout de même réfractaire. En effet, s'il n'est plus exprimé avec la vigueur des temps

18. Athanase, Vie d'Antoine, ouvr. cité, p. 92-93.

19. Évangile de Matthieu, XIX, 2I. La Bible, ouvr. cité, p. 64. 
de persécution, le procès de la société est permanent, ne serait-ce que par le choix de vie de l'ermite, qui nie au modèle du siècle toute légitimité à encadrer la quête spirituelle. Le comportement des ermites prit rapidement une dimension collective, qui invite à interroger les relations entre individus face aux structures du pouvoir. Cette question occupa un temps Roland Barthes, notamment quand, dans Comment vivre ensemble, il propose comme principe de l'équilibre des communautés un fonctionnement collégial qui implique une vision négative du pouvoir ${ }^{20}$. Autour d'Antoine, puis de Pacôme et de Macaire l'Ancien, se fondèrent des communautés, de tailles variables, allant d'un ou deux disciples à des dizaines de moines. Comme le rappelle justement Roland Barthes, elles nécessitent une forme d'idiorythmie, une faculté à vivre ensemble sans s'aliéner à l'autre ni l'aliéner, et ce, compte tenu du fait, qui complexifie singulièrement le problème posé, que la quête spirituelle, au désert, est elle-même à la fois recherche de personnalisation du rapport à Dieu et recherche d'une forme de «désindividuation» face à lui. Les deux dimensions de l'aspect politique du mythe sont alors posées : l'ermite, puis le moine, respecte la hiérarchie humaine, mais prend néanmoins le parti d'une recherche utopique de vie communautaire, et, ce faisant, approfondit l'expérience au point d'évacuer la dimension interpersonnelle du groupe, pour qu'il ne tende que vers Dieu. L'utopie politique, sociale, du moine, est donc celle de la recherche d'une triple altérité : communion à l'altérité du groupe, recherche en soi de l'altérité de l'âme pour qu'elle soit, par le silence et la prière, mise en relation avec l'altérité absolue de Dieu.

Ainsi s'esquisse déjà le mythe qui entoure la naissance du monachisme, dans les pratiques des premiers ermites qui se dirigent de façon relativement empirique vers le cénobitisme ou le monachisme. Cette utopie communautaire a nourri de multiples penseurs, des docteurs de l'Église et Benoît de Nursie à Port-Royal, qui redécouvre les Pères grâce aux travaux d'Arnauld d'Andilly, jusqu'à pénétrer si fortement les structures de notre civilisation qu'elle participe de recherches aussi variées que celles de Roland Barthes ou même de certains mouvements hippies, du New Age au «renouveau charismatique» de certaines églises. L'utopie des Pères, du point de vue social, cristallise une tension dont la dynamique offre à leur histoire une fonction mythique, qui pousse à penser, réévaluer sans cesse les modèles sociaux, comme c'est encore le cas, par exemple, dans l'utopie millénaire qui anime la République monastique du mont Athos.

20. R. Barthes, Comment viure ensemble, ouvr. cité, p. 69. 


\section{Du bon usage de la connaissance au désert du langage}

L'autre aspect des activités d'Antoine intéressant du point de vue du mythe à venir est celui de sa prédication. En effet, elle pose les termes qui sont devenus définitoires d'une forme de "mythe de la connaissance" dans les traditions chrétiennes. Nous l'avons mentionné, bien qu'il aspire à la solitude, Antoine ne refuse pas de recevoir des visites, et prend la parole quand on le sollicite : au désert, devant ses visiteurs et ses disciples de plus en plus nombreux, en Alexandrie, pendant les persécutions et les débats qui divisent la communauté face aux thèses d'Arius. Le silence de ses solitudes nourrit la parole de ses prédications : il ne faut pas céder aux charmes de l'intelligence, la vérité du christianisme ne s'entoure pas de démonstrations mais entre en rupture avec la rhétorique des philosophes, des polythéistes et des hérésiarques. On est bien loin, alors, de Thomas d'Aquin. Lors d'une controverse avec des philosophes et des prêtres grecs de cultes polythéistes, Antoine affirme :

Vous êtes au comble de l'incrédulité en cherchant des raisonnements, des discours, mais nous, ce n'est pas dans le langage persuasif de la sagesse (I Cor., II, 4), grecque que nous démontrons. Nous persuadons par la foi qui renverse l'armature des discours ${ }^{21}$.

L'ermite, une fois encore, obéit aux injonctions de l'apôtre Paul :

Prenez garde qu'on ne vous emporte avec la philosophie, ce vain leurre qui s'inspire de la tradition humaine et des éléments du monde, mais non du Christ ${ }^{22}$.

La philosophie, dans cette occurrence paulinienne, prend le sens des traditions religieuses antiques, autant que des raisonnements systématiques des philosophes, au sens où nous entendons aujourd'hui ce terme, comme le précise le traducteur ${ }^{23}$. Ainsi, tant les connaissances et les exégèses sapientiales des religieux juifs et polythéistes que celles des philosophes et des savants sont impropres à mener vers la "connaissance" authentique, celle de Dieu par la révélation de son christ. Cela a deux effets : le raisonnement est dépouillé de sa fonction herméneutique pour devenir la structure qui donne à l'illusion son apparente vraisemblance, et la parole elle-même se distingue des stratégies discursives pour devenir une dynamique de performation, et non d'explication. Antoine se méfie autant des connaissances et du savoir qu'il valorise une parole spécifiquement vouée à «toucher» l'homme, à l'émouvoir au sens premier du terme de «mise en mouvement». Parole performative puisqu'elle

2I. Athanase, Vie d'Antoine, ouvr. cité, p. 9I.

22. Voir l'Épître aux Colossiens, II, 8. La Bible, ouvr. cité, p. 665.

23. Dans le cas présent, M. Léturmy. 
crée en l'homme non une connaissance mais un désir de vivre en relation avec la divinité : la parole révélée de la Bible comme celle de la prière doit engager l'homme au-delà de son intelligence, l'en dépouiller même pour mettre en branle, en lui, le principe spirituel, l'âme.

C'est bien là que le texte d'Athanase, rejoint par celui de Jérôme, met en place les thèmes qui, dans les traditions chrétiennes, firent des gestes antonines et pauliniennes les fondations d'un mythe épistémologique. Plus précisément, il s'agit, pour Athanase et Jérôme, de montrer que la connaissance exige un usage éthique et, par extension, que l'intransigeance des ermites dans leur ascèse intellectuelle annule les connaissances. D'ailleurs, si cela n'est pas explicite dans le texte d'Athanase, les traditions orales et hagiographiques postérieures décrivent régulièrement Antoine comme peu éduqué, sinon illettré. Bien que ce soit peu probable du fait de ses origines sociales, il n'est pas impossible, dans un contexte où la transmission orale jouait un rôle prépondérant, que l'ermite ait acquis sa connaissance de la Bible par l'écoute des lectures et des prédications faites dans les églises.

En ce qui concerne Paul de Thèbes, personnage dont il est de plus en plus communément admis qu'il est fictionnel, il est remarquable que Jérôme, son «biographe», tienne à préciser qu'il avait "une connaissance hors pair des lettres grecques et égyptiennes, le cœur bon, et un grand amour de Dieu $»^{24}$. Jérôme, grand lettré s'il en fut, renouvelle par ces précisions le topos biblique selon lequel la meilleure connaissance ne vient pas de l'érudition, mais de l'attitude intérieure. Il approfondit, en quelque sorte, la fameuse béatitude des "pauvres en esprit»; c'est aussi l'interprétation métaphorique qu'un intellectuel peut faire de cette parole de l'évangile de Marc:

Rien de ce qui est extérieur à l'homme et qui entre en lui ne peut le profaner; mais c'est ce qui sort de l'homme qui profane l'homme ${ }^{25}$.

Nous évoquions un "usage éthique» de la connaissance : davantage encore qu'Athanase, volontiers politique et polémique dans les discours qu'il prête à Antoine, Jérôme met en place un système de valeurs, une forme de déontologie, avec sa Vie de Paul de Thèbes. Contrairement à Athanase qui avait à composer avec le caractère d'Antoine, qu'il connaissait personnellement, Jérôme peut à son gré insister sur les caractéristiques qui l'intéressent, notamment le «bon usage» de l'érudition. Si Athanase, à partir de la vie réelle d'Antoine, pratique une prédication qui inscrit l'ermite dans des problématiques à tendances universalistes, en particulier celle d'un dogme universel dépassant les particularismes des communautés, Jérôme ne s'embarrasse pas d'historicité : il

24. Jérôme, Vie de Paul, ouvr. cité, p. 28.

25. Évangile de Marc, VII, I5. La Bible, ouvr. cité, p. I30-I3I. 
fonde une fiction, une fiction de la connaissance, qui contribua notablement à l'établissement de valeurs, mais aussi à la tradition des auteurs mystiques.

Ainsi, dans la lignée des prophètes au désert, que nous mentionnions plus haut, les ermites égyptiens fondent un rapport particulier au langage et à la connaissance, dont les effets, dans la littérature, sont considérables. L'alternance entre silence et parole, silence intérieur, parole impersonnelle de la prière du $\operatorname{cour}^{26}$, représente le moyen par excellence pour arriver à l'idéal spirituel : la relation permanente à Dieu. En effet, environné des signes trompeurs envoyés par le démon, que ce soient des visions ou les illusions de l'intelligence, l'ermite pose la question de la validité du signe. Il y répond partiellement en dépouillant la parole de sa fonction herméneutique pour la ramener à sa dynamique signifiante, la mise en présence des instances parlantes, refusant au langage toute autre efficience que celle de la relation. Débat éminemment littéraire auquel prennent part, à la suite des Pères et en marge des dogmes et des écoles, les auteurs mystiques, du Pseudo-Denys à Ruysbroeck et jusqu’à, plus près de nous, Marie de la Trinité. Enfin, les Pères participent significativement au modèle culturel du sage, voire du moraliste, dont les paroles mesurées représentent la verticalité de la relation à la sagesse, qu'on la nomme Dieu ou non, en ce que, pour paraphraser Isaïe, la parole tombe, et ne se retire pas sans effet ${ }^{27}$.

En guise de conclusion partielle, peut-on oser dire que la littérature, de son côté, agit de manière comparable, qu'elle soit ou non spirituelle? Rimbaud, Michaux, Lorand Gaspar, pour ne citer que trois poètes liés à ces thèmes, ont tous eu maille à partir avec le désert, physique, mais aussi le désert de la langue, le désert du signifiant qui, par sa dynamique, dépouille le langage de tout sens fixe, de toute certitude, tout comme l'ermite, par sa discipline spirituelle, se voyait sans cesse dépouillé du discours, de sa personnalité, et même de sa foi. Naturellement, il n'est pas question de ramener la question du rapport entre silence et langage uniquement aux Pères du désert. La crise du signe qui traverse la littérature du tournant de la modernité a des origines multiples, au rang desquelles on oublie peut-être trop souvent de compter l'intuition personnelle du poète. Mais il est bon de se rappeler que les Pères

26. La prière du cœur est une pratique spirituelle toujours très recherchée par les moines, en particulier des diverses églises orthodoxes. Elle consiste en la répétition, dans l'idéal permanente, de la phrase : "Jésus Christ, fils de Dieu, prends pitié de moi ", avec, parfois, quelques variantes. Attribuée à l'origine aux Pères du désert, elle est au centre de l'imposant corpus qui constitue la Philocalie des orthodoxes. Voir par exemple Petite Philocalie de la prière du cour, éd. et trad. de J. Gouillard, Seuil, 1979.

27. Isaïe, LV, IO-II. Voir par exemple : La Bible de Jérusalem, Desclée de Brouwer, 1975, p. 1182. 
du désert ont structuré en partie un débat permanent, au moins jusqu'à la récente sécularisation de la culture occidentale.

Référents longtemps essentiels, à la base d'un mythe culturel, un mythe de culture, les Pères du désert, parfois un peu oubliés, apportent une contribution originale au débat littéraire. À la question toujours posée : comment faire vivre ensemble les hommes? Les Pères répondent par un ensemble de tentatives empiriques qui alimentent l'imaginaire social, sans que les Règles qui ont découlé de leurs expériences n'apportent jamais une réponse véritablement pérenne et, de ce fait, fermée. À la question elle aussi toujours posée : quelle parole est juste, quelle vérité possible? Les Pères répondent, comme cet autre ermite, abba Théodore, à un frère qui lui demande de le guider : "Je suis moimême en péril, que pourrais-je te dire ${ }^{28}$ ". Les Pères du désert, s'ils prêchent parfois, nous renvoient d'abord à notre propre silence, nous donnent, plutôt que des réponses dogmatiques, des outils, et un désir. En cela, peut-être que les Pères du désert sont à compter parmi les religieux les plus littéraires de notre histoire.

28. Abba, dis-moi une parole, éd. et trad. de L. Regnault, Sablé-sur-Sarthe, abbaye Saint-Pierre de Solesmes, I998, p. 8I. 